\title{
Editorials
}

\section{Cardiovascular surgery as a single specialty: The case to unify cardiac and vascular surgery}

\author{
Charles Stewart Roberts, MD
}

From the Section of Thoracic and Cardiovascular Surgery, The Heart and Vascular Center, Winchester Medical Center, Valley Health System, Winchester, Va.

Received for publication Feb 29, 2008; accepted for publication April 17, 2008

Address for reprints: Charles Stewart Roberts, MD, 190 Campus Blvd, Suite 410, Winchester, VA 22601 (E-mail: croberts@ valleyhealthlink.com).

J Thorac Cardiovasc Surg 2008;136:267-70 0022-5223/ $\$ 34.00$

Copyright (C) 2008 by The American Association for Thoracic Surgery

doi:10.1016/j.jtcvs.2008.04.010
$\mathrm{C}$ ardiovascular surgery came of age in the 20th century. Carrel, ${ }^{1,2}$ in the first decade, described an end-to-end suture technique to connect blood vessels, which launched vascular surgery. Gibbon ${ }^{3}$ in 1953 first used a heart-lung machine clinically, which launched cardiac surgery. Cardiovascular deaths in the United States have led all others every year of the 20th century except 1918, the year of the influenza epidemic.

By the end of the 20th century, cardiovascular surgery was formally separated into two different disciplines, cardiac surgery and vascular surgery. Cardiac surgery is paired with general thoracic surgery as part of the specialty, thoracic surgery, which became a primary board specialty in 1971. Vascular surgery more recently emerged as a surgical specialty, first by establishing its own residency programs and later by gaining approval for a primary certificate in vascular surgery by the American Board of Surgery. ${ }^{4}$ Both cardiac and vascular surgery address disorders of the circulation. In my opinion, they are unnaturally separated and should be unified into one specialty. Here's why:

The organ system is the best determinant of a surgical specialty, not a region of the body such as the thorax or the abdomen. William Harvey, in his 1628 book on the movement of the heart and blood, described arteries, veins, and the heart as a system. ${ }^{5}$ Harvey used the term circle and it variants (circulation, circulate, circular, circuit) 27 times in the last 10 chapters (none in the first 7). It seems incongruous today to partition the circle, to have one specialty treat one part of the circulation and another specialty treat another part of the circulation, depending on its position in relation to the diaphragm or clavicle or whether the heart lung machine is necessary.

Cardiovascular is a better term to describe a surgical specialty than cardiothoracic. Whereas the term cardiovascular defines an organ system, the term cardiothoracic describes a single organ and a region of the body. A parallel term, esophagothoracic, for example, is not used. The confusion is heightened because cardiothoracic surgery, although it conveniently describes many surgical units in the United States, is not the formal term used to describe the current specialty, which is thoracic surgery.

A lifelong study of the circulation is required in both cardiac and vascular surgery. MedicineWorld.Org provides a list of 125 journals under the heading of "cardiology". It lists 187 under the heading of "cancer." No single surgeon can stay current in both cardiovascular disease and neoplastic disease. A cardiovascular surgeon, in contrast to a thoracic surgeon, would be able to focus on the circulatory system and its diseases, which is sufficient for one career.

The dominant disease treated by cardiac and vascular surgeons is the same, namely, atherosclerosis. It is the same disease, of course, in the coronary, carotid, and femoral arteries. ${ }^{6}$ Although operative treatment of a single arterial bed may vary in technical ways, systemic treatment of atherosclerosis is virtually identical for coronary artery disease and peripheral arterial disease.

A surgeon familiar with coronary atherosclerosis is well suited to treat carotid or femoral atherosclerosis. Performance of a coronary artery bypass grafting (CABG) 


\section{Abbreviations and Acronyms}

AATS $=$ American Association for Thoracic Surgery

STS = Society of Thoracic Surgeons

$\mathrm{CABG}=$ coronary artery bypass grafting

procedure in the morning and a lobectomy in the afternoon is certainly an everyday occurrence in the United States, but a far more natural sequence is to follow the CABG with a carotid endarterectomy or a femoropopliteal bypass or repair of an abdominal aortic aneurysm.

The same surgeon can treat the single patient with atherosclerosis in different arterial beds. Many of my patients who have undergone $\mathrm{CABG}$ have required carotid endarterectomy or femoropopliteal bypass and vice versa. Knowledge of the consequences of atherosclerosis in one arterial bed is useful in the treatment of atherosclerosis in another arterial bed. For example, an expertise in carotid atherosclerosis is useful in the evaluation of a patient with concomitant coronary atherosclerosis and vice versa. Whereas operations to treat aneurysms tend to be curative, operations to treat blockages are palliative. Atherosclerotic patients keep coming back, some presenting first with coronary artery disease, others presenting with peripheral arterial disease, and many presenting with both.

Aneurysms and dissections of the aorta and its branches, no matter what location, can be treated by the same surgeon. Endovascular repair of aortic aneurysms has been developed by vascular surgeons and many now treat thoracic aortic aneurysms. Thoracic surgeons also perform this procedure now, however, and the overlap is obviously unnecessary. The marriage of cardiac and vascular surgeons would eliminate overlap or barriers in the treatment of aortic disease by open or closed techniques. Hybrid procedures, involving combined endovascular and open techniques, inside or outside of the chest, become less complicated when the treating surgeon is not limited to an anatomic region.

The surgical techniques necessary in both coronary and noncoronary arterial surgery are similar. Operating on arteries inside and outside the thoracic cage involves similar techniques-proximal and distal control, use of autologous and nonautologous conduits, vascular anastomoses, patch or primary closures, antegrade and retrograde flushing, avoidance of end-organ ischemia and embolization, anticoagulation and its reversal, and so on. These standard vascular methods are currently learned by both vascular and thoracic surgeons but are intended to be applied only outside the chest by vascular surgeons and inside the chest by thoracic surgeons.

Cardiovascular surgeons may benefit from the combination of open cardiac and vascular cases. The rise of closed procedures on the aorta and all of its branches has meant fewer open procedures. The number of CABG procedures, in particular, has declined in recent years. The same is true for open revascularization procedures for the lower extremities.
The implications for cardiac and vascular surgeons and patients are profound if one considers the inverse relation of surgical volume and adverse outcome. Performing fewer CABG procedures yearly, for example, may be concerning if the other operations in one's case mix are video-assisted thoracic surgery, mediastinoscopy, lobectomy, decortication, and esophagectomy. However, if the other operations are open vascular procedures, such as carotid endarterectomy, and various peripheral arterial operations, then the technical requirements overlap with $\mathrm{CABG}$-fewer $\mathrm{CABG}$ procedures annually may not spell disaster.

Vascular surgeons, perhaps more so than cardiac surgeons, feel the pull of the angiography suite and endovascular therapy. This evolution, however, diminishes considerably the vascular surgeon's experience in open procedures. For the vascular surgeon whose bent is open procedures, adding $\mathrm{CABG}$ procedures to the practice may enhance his or her skill in open operations.

A cardiovascular surgery specialty is historically fitting. One of the first monograms on this subject, Surgery of the Vascular System, was published in 1913 by Bertram M. Bernheim $^{7}$ of Baltimore, who was first in the United States (1915) to interpose an autologous vein graft after resecting a popliteal aneurysm. ${ }^{8}$ The book contains chapters on general technique, transfusion, end-to-end suture, lateral anastomosis, transplantation of a segment of vein or artery, arteriovenous anastomosis (reversal of the circulation), varicose veins, and aneurysms, with exquisite illustrations. Chapter 13 is called "Surgery of the Heart," the point being that cardiac surgery was originally considered to be within the field of vascular surgery. It was part of "the field of blood-vessel surgery."

Early pediatric cardiovascular operations were purely vascular. In 1938 Gross ligated a patent ductus arteriosus, ${ }^{9}$ and in 1944, Blalock constructed a subclavian-pulmonary anastomosis for a patient with the tetralogy of Fallot. ${ }^{10}$ Other common pediatric cardiovascular operations are purely vascular, including repair of coarctation of the aorta.

Many early cardiac surgeons began as general surgeons, thoracic surgeons, and vascular surgeons, and many became cardiovascular surgeons, contributing in both cardiac and vascular arenas. Michael E. DeBakey was the founding editor of the Journal of Vascular Surgery. His original contributions to the surgical treatment of aneurysms and atherosclerosis in multiple arterial beds were numerous. ${ }^{11,12}$ His surgical creativity was not limited by the diaphragm or the clavicle or the heart-lung machine. In an interview in 1997, he remarked: "The cardiac and vascular services are separated in many places. I object to that for the simple reason that I consider the cardiovascular system a unified system." 13

Denton A. Cooley, known for his numerous contributions to the treatment of congenital and acquired cardiovascular disease, performed the first successful carotid endarterectomy in $1956 .{ }^{14}$ His book with Wukasch, Techniques in Vascular Surgery, was my guide when developing a practice 
of vascular surgery. ${ }^{15}$ His namesake society is called the Denton A. Cooley Cardiovascular Surgical Society. The term used is cardiovascular - not cardiac or vascular or thoracic or cardiothoracic.

The specialty of thoracic surgery was founded on general thoracic surgery, not cardiovascular disease. The focus early in the 20th century was on the operative treatment of pulmonary infections, particularly tuberculosis. Lung cancer before 1930 was infrequent, and cardiovascular operations inside the chest were virtually nonexistent.

In his 1953 book, A Practice of Thoracic Surgery, A. L. d'Abreu ${ }^{16}$ of Birmingham, England, wrote, "In 1934, largely through the kind encouragement of the late Professor Lyle Cummins, the Professor of Tuberculosis in the University of Wales, I started the study and practice of thoracic surgery." One did not enter thoracic surgery at that time to treat cardiovascular disease. His book contains 27 chapters, only 5 of which pertain to the cardiovascular system (2 diagnostic and 3 surgical), representing $18 \%$ of the book by pages. General thoracic surgery dominates, with sections on bronchopulmonary anatomy, pyogenic infection, pulmonary tuberculosis, diseases of the esophagus, and diaphragmatic problems. D'Abreu's book appeared the year the heartlung machine was first introduced clinically.

Also in 1953, Lindskog and Liebow ${ }^{17}$ published Thoracic Surgery and Related Pathology, another book dominated by infectious and neoplastic diseases. The follow-up book appeared in 1962 with a title change, now called Thoracic and Cardiovascular Surgery with Related Pathology, with the contribution of the cardiovascular surgeon, William Glenn. ${ }^{18}$ This new version had two parts: thoracic surgery, which contained 28 chapters (580 pages), and cardiovascular surgery, which contained 21 chapters (444 pages) - two completely different fields of study. This was the beginning of the complicated marriage of cardiovascular surgery and general surgery inside the chest.

The major surgical societies currently do not completely serve the many surgeons who practice cardiovascular surgery. The dominant societies segregate cardiac and vascular surgeons. In the annual meetings of The American Association for Thoracic Surgery (AATS) and The Society of Thoracic Surgeons (STS), peripheral vascular surgery is not part of the program, and a significant portion of both meetings is devoted to general thoracic surgery. The Society for Vascular Surgery does not address the surgical treatment of the first branches of the aorta (coronary arteries) and gives probably inadequate attention to intrathoracic vascular surgery.

The AATS was founded in 1917 on general thoracic surgery, before the advent of antimicrobial therapy. The Journal of Thoracic Surgery, the official journal of the AATS, was first published in 1931, and none of the 6 original articles in the first issue concerned cardiovascular surgery. By 1959, the Association was faced with the rapid progress of cardiovascular surgery, and it changed the name of its publication to The Journal of Thoracic and Cardiovascular Surgery. ${ }^{19}$ It currently divides its subject matter between intrathoracic cardiovascular surgery and general thoracic surgery, having abandoned peripheral vascular surgery altogether. In the February 2008 issue of The Journal of Thoracic and Cardiovascular Surgery, the 41 articles included 33 on intrathoracic cardiovascular conditions ( 1 on thoracoabdominal aortic aneurysm) and 8 on general thoracic conditions.

The STS was founded in 1963. On its emblem, adopted in 1965, there were four drawings - a heart, an esophagus, the lungs, and the abdominal aorta. The emblem was changed in 1994 to reflect the abandonment of extrathoracic vascular surgery. Now there appear four drawings - the heart, the lungs, three structures together (the thoracic aorta, the trachea, and the esophagus), and the thoracic cage. This change is certainly understandable, given the Society's focus on thoracic surgery, but it clearly partitions the cardiovascular system. Sidney Levitsky, ${ }^{20}$ in his presidential address to the STS in 2006, reflected on the change: "The peripheral vascular portion of the STS logo, exemplified by the abdominal aortic aneurysm, was changed in 1994 to the thoracic aorta as cardiac surgeons turned away from peripheral vascular surgery and focused their attention on coronary artery surgery...Both specialties and our patients would benefit, if reunification occurred."

The official journal of the STS, The Annals of Thoracic Surgery, first published in 1964, also has a division in its subject matter across four organ systems - the cardiovascular system, the respiratory system, the alimentary tract, and the musculoskeletal system. In simplistic terms, of course, it has two subjects, cardiovascular surgery within the chest and general thoracic surgery. In the February 2008 issue, the 40 "original articles" are neatly separated under two different headings: 10 articles appeared under "general thoracic" and 30 under "cardiovascular." No article addressed extrathoracic vascular surgery.

The Society for Vascular Surgery was founded in 1947. In the February 2008 issue of its Journal of Vascular Surgery, 3 of the 25 articles under the first section, "Clinical Research," addressed intrathoracic vascular conditions. The lead article was on thoracic endovascular aneurysm repair, and the published presidential address was on carotid stenting, which reflect the clinical range encompassed now by the specialty.

In my own practice, which consisted of 340 operations in 2007, the two most common operations are CABG and carotid endarterectomy, which are the two most common operations in thoracic and vascular surgery, respectively. This cardiovascular surgical practice, however, requires two different national society meetings, two different specialty journals, and two different Web sites (CTSNet and VascularWeb) to keep abreast.

The concept of the "Heart and Vascular Center" or "Cardiovascular Institute" makes sense and fits well with a "cardiovascular" surgical specialty and service line. One does not see the "Heart and Esophageal Center" or the "Lung 
and Vascular Center." Cardiac and vascular surgeons are already co-located in these cardiovascular centers. A cardiovascular surgeon would be well suited to provide all surgical services in a "Heart and Vascular Center."

A single cardiovascular surgical specialty may enhance relations with other specialties. Cardiologists have no selfimposed barriers, such as the diaphragm and the clavicle. Their realm now is the entire circulatory system. A cardiovascular surgeon may be of the same mind. The referring cardiologist or primary care physician need not inquire whether the thoracic surgeon or the vascular surgeon does this or that operation.

Vascular surgeons attract a considerable number of referrals directly from internists, family practitioners, podiatrists, and others. This position of actually evaluating and treating patients with atherosclerosis or aneurysms is different from the usual position of the thoracic surgeon, who is referred a patient for an operation after a rather thorough evaluation by a cardiologist. After performing a cardiovascular operation in the chest, such as CABG, the thoracic surgeon usually relinquishes the patient by 2 months postoperatively. Vascular surgeons, in contrast, make most clinical decisions about the treatment of noncoronary arterial disease in their patients. As a cardiovascular surgeon, I refer numerous patients with atherosclerosis to cardiologists for cardiac studies, particularly in preparation for noncoronary arterial operations. The relationship with cardiologists, therefore, achieves better balance.

A unification of cardiac and vascular surgery would bring all open and closed cardiovascular operations to bear in a single specialty. Vascular surgeons already are comfortable in the angiographic suite. Cardiac surgeons are slowly adopting more closed procedures. Expertise in closed and hybrid procedures may be a requirement for a viable cardiovascular surgical practice in the future. A merger of cardiac and vascular surgery may facilitate the free flow of ideas and techniques. Numerous closed cardiac procedures such as percutaneous aortic valve insertion and percutaneous mitral valve repair are gaining a foothold, and the combined expertise of cardiac and vascular surgeons might bring more progress.

Cardiac surgeons who are part-time cancer surgeons may serve the cancer patients less well than full-time general thoracic surgeons. Cardiovascular and cancer conferences are entirely different. Cardiovascular conferences involve cardiologists and interventional radiologists, all discussing diseases of the circulatory system, usually atherosclerosis. Cancer conferences involve medical oncologists, surgical oncologists, radiation oncologists, and specialty radiologists (eg, mammogram experts), all discussing tumors. Virtually no overlap of participation occurs in cancer and cardiovascular conferences, except by thoracic surgeons. This duality seems out of step with postmillenium health care delivery.

A cardiovascular surgical residency may attract top trainees. In the 2008 match for thoracic surgery, 96 residents applied for 130 positions and some 81 (67\%) positions were filled. ${ }^{21}$ For vascular surgery, 139 residents applied for 119 positions and some $115(97 \%)$ positions were filled. ${ }^{22}$ One may conclude that medical students and residents have minimal interest now in both thoracic surgery and vascular surgery as separate surgical specialties. Furthermore, the training positions are secured with minimal competition$91 \%$ of thoracic surgery applicants matched and $83 \%$ of vascular surgery applicants matched. ${ }^{21,22}$

The unification of cardiac and vascular surgery into one residency training program, in my opinion, would be a win-win proposition. A unified cardiovascular surgical training program not only would attract more applicants but also would promote more keen competition for positions, and the final product would be more valuable.

\section{References}

1. Carrel A. Surgical technique for vascular anastomoses and organ transplants [French]. Lyon Med. 1902;98:859-63.

2. Carrel A. The surgery of blood vessels. Johns Hopkins Hosp Bull. 1907; $18: 18$.

3. Gibbon JH Jr. Application of a mechanical heart and lung apparatus to cardiac surgery. Minn Med. 1954;37:171-80.

4. Cronenwett JL, Liapis CD. Vascular surgery training and certification: an international perspective. J Vasc Surg. 2007;46:621-9.

5. Harvey W. Exercitatio anatomica de motu cordis et sanquinis in animalibus. 4th ed. (Translated by Chauncey D. Leake.) Springfield [IL]: Charles C Thomas Publisher; 1958.

6. Roberts WC. Atherosclerosis: its cause and its prevention. Am J Cardiol. 2006;98:1550-5.

7. Bernheim BM. Surgery of the vascular system. Philadelphia: J.B. Lippincott; 1913.

8. Bernheim BM. The ideal operation for aneurysms of the extremity: report of a case. Johns Hopkins Hosp Bull. 1916;27:1.

9. Gross RE, Hubbard JP. Surgical ligation of patent ductus arteriosus. JAMA. 1939;112:729-31.

10. Blalock A, Taussig HB. The surgical treatment of malformations of the heart in which there is pulmonary stenosis or pulmonary atresia. JAMA. 1945;128:189-202.

11. DeBakey ME. The development of vascular surgery. Am J Surg. 1979; 137:697-738.

12. DeBakey ME, Lawrie GM, Glaeser DH. Patterns of atherosclerosis and their surgical significance. Ann Surg. 1985;201:115-31.

13. DeBakey MD, Roberts WC. Michael Ellis DeBakey: a conversation with the editor. Am J Cardiol. 1997;79:929-50.

14. Al-Naaman YD, Carton CA, Cooley DA. Surgical treatment of arteriosclerotic occlusion of common carotid artery. J Neurosurg. 1956;13: 500-6.

15. Cooley DA, Wukasch DC. Techniques in vascular surgery. Philadelphia: W.B. Saunders; 1979.

16. D'Abreu AL. A practice of thoracic surgery. London: Edward Arnold \& Co; 1953.

17. Lindskog GE, Liebow AA. Thoracic surgery and related pathology. New York: Appleton-Century-Crofts; 1953.

18. Lindskog GE, Liebow AA, Glenn WWL. Thoracic and cardiovascular surgery with related pathology. New York: Appleton-Century-Crofts; 1962.

19. Ferguson TB. The Journal of Thoracic and Cardiovascular Surgery diamond jubilee-a reflection. J Thorac Cardiovasc Surg. 2006;132:451-2.

20. Levitsky S. Navigating the new "flat world" of cardiothoracic surgery. Ann Thorac Surg. 2007;83:361-9.

21. Vital Signs. Thoracic surgery fellowship 2008 matches. Thoracic Surgery News. 2007;3(5):1.

22. Vital Signs. Vascular surgery fellowship 2008 matches. Vascular Specialist. 2007;3(5):1. 